\title{
AEO-7 surfactant is "super toxic" and induces severe cardiac, liver and locomotion damage in zebrafish embryos
}

\author{
Maha Al-Asmakh ${ }^{1,2}$, Amin F. Majdalawieh ${ }^{3}$, Aboubakr M. Abdullah ${ }^{4,5}$, Nadin Younes ${ }^{1}$, Sahar I. Da'as ${ }^{6}$, \\ A. Bahgat Radwann ${ }^{4}$, Mostafa H. Sliem ${ }^{4}$, Houria Ech-Cherif ${ }^{1}$, Gianfranco Pintus ${ }^{7}$ and Gheyath K. Nasrallah ${ }^{1,2^{*}}$ (D)
}

\begin{abstract}
Background: Fatty alcohol polyoxyethylene ether-7 (AEO-7), a non-ionic surfactant, has recently been receiving extensive attention from the ocean pipeline industry for its ability to inhibit corrosion. However, the present lack of information concerning the potential environmental toxicity of AEO-7, especially towards aquatic organisms, is a major impediment to its wider application. Here, we assess potential adverse effects of AEO-7 on zebrafish embryos employing a variety of assays, including (i) a mortality/survival assay which allowed the median lethal concentration $\left(\mathrm{LC}_{50}\right)$ to be calculated; (ii) a teratogenicity assay on the basis of which the no observed effect concentration (NOEC) was determined; and (iii) specific assays of cardiotoxicity, neurotoxicity (based on locomotion), hematopoietic toxicity (the level of hemoglobin as revealed by o-dianisidine staining) and hepatotoxicity (liver steatosis and yolk retention examined by staining with Oil Red O).

Results: AEO-7 caused mortality with a calculated $L_{50}$ of $15.35 \mu \mathrm{g} / \mathrm{L}$, which, according to the U.S. Fish and Wildlife Service (USFWS) Acute Toxicity Rating scale, should be considered "super toxic". Although at its NOEC (0.8 $\mu \mathrm{g} / \mathrm{L})$, there were no signs of significant teratogenicity, cardiotoxicity, or hemopoiesis toxicity, $3.2 \mu \mathrm{g} / \mathrm{L}$ AEO-7 exerted dramatic detrimental effects on organ development.
\end{abstract}

Conclusion: On the basis of these findings, we recommend that the industrial usage and environmental impact of AEO-7 be re-evaluated and strictly monitored by environmental and public health organizations.

Keywords: AEO-7 surfactant, Cardiotoxicity, Neurotoxicity, Hepatotoxicity, Zebrafish embryos

\section{Introduction}

Surfactants have attracted wide attention due to their unique physio-chemical properties and their abilities to be tailor-made to suit various applications in comparison with conventional solvents [1-4]. Surfactants are widely used in the industry of detergents and other household products such as hair conditioners and personal care products. In addition, surfactants have attracted

\footnotetext{
*Correspondence: gheyath.nasrallah@qu.edu.qa

${ }^{2}$ Department of Biomedical Sciences, College of Health Sciences, QU Health, Qatar University, Women's Science building, C01, P.O. Box 2713, Doha, Qatar

Full list of author information is available at the end of the article
}

considerable commercial interest [2, 4], especially in connection with many fundamental industrial applications such as petroleum oil recovery, and most importantly, inhibition of corrosion [4], as it remains challenging to develop "green", environmentally friendly, organic corrosion inhibitors that are still cost-effective [5]. As a result, the total worldwide production of surfactants is continuously growing reaching 14.09 million tons in 2017, and is expected to increase to over 24 million tons annually by 2020 [6, 7]. The 2019 global market for surfactants, worth an estimated $\$ 39$ billion, is expected to grow at $2.6 \%$ per annum over the next five years to reach $\$ 46$ billion by 2024 [8]. Following the use of surfactants in industrial 
and household usage, residual components are dispensed into sewage systems or directly into surface waters, most of which end up dispersed into various environmental pools such as water, soil, or sediment, therefore, disrupting the water cycles in the ecosystem. Additionally, surfactants can accumulate in living organisms leading to unknown potential toxic effects $[9,10]$.

The toxicity of surfactants has been characterized to some extent [11-17] and these compounds are often considered the best "green" inhibitors of corrosion [4, 18-24]. However, their potential toxic effects, towards aquatic organisms, remain a concern $[9,10,25]$. For instance, Vaughan and his colleagues studied the acute toxicity of 15 different anionic, cationic and non-ionic surfactants on zebrafish embryos and adults. They reported that embryos are as sensitive to cationic and non-ionic surfactants as adult fish, but may be more sensitive to anionic surfactants compared to adult fish [17]. In addition, Wang et al. exposed zebrafish embryos to three commonly employed surfactants (sodium dodecyl sulfate, dodecyl dimethyl benzyl ammonium chloride, and fatty alcohol polyoxyethylene ether), two of these proved to be highly toxic at concentrations as low as $1 \mu \mathrm{g} / \mathrm{mL}$ [26]. Moreover, both anionic and, in particular, non-ionic surfactants were highly toxic to various aquatic fauna [27]. Therefore, the environmental friendliness of many of these widely used compounds needs to be reconsidered in order to be able to select the least toxic and most biodegradable surfactants for commercial use.

Fatty alcohol polyoxyethylene ethers (AEOs) are the largest and most rapidly expanding family of non-ionic surfactants. Many researchers believe that AEOs consumption will continue to grow and will become the leading household detergents [28]. Fatty alcohol polyoxyethylene ether-7 (AEO-7) is a commercial product that is commonly utilized as an emulsifier to prepare oil-in-water $(\mathrm{O} / \mathrm{W})$ type emulsion. In addition, it is generally used as household detergent, solubilizing agent, spreading agent, wetting agent, foaming agent, dispersing agents, penetrating agents, and textile auxiliary's chemical. In addition, it is used as a wool detergent and fabric scouring agent in the wool spinning industry. Furthermore, our collaborators have recently demonstrated that AEO-7 acts as a highly efficient "potentially green" inhibitor of corrosion, even under extremely harsh conditions [21]. Even though AEO-7 is widely used in many "green" application, the presence of this compound, even at concentrations as low as $1 \mathrm{mg} / \mathrm{L}$, can result in the formation of a persistent foam on wastewater, which attenuates the exchange of gas and causes the water to stink [29]. Furthermore, high concentrations of AEOs in wastewater not only kill microorganisms and inhibit the degradation of other toxic substances, but also reduce the level of dissolved oxygen [29]. Interestingly, according to patents published by AEO-7 manufacturers, AEO-7 is considered safe to be used as a detergent. However, the method of toxicity evaluation for this compound was not described [30-33]. Moreover, until now, only one study explored the potential toxic effect of AEO-7 on zebrafish embryos and was limited to analyzing the effect of AEO-7 on gene expression and behavior of the embryos [34]. However, the authors did not study other important parameters of toxicity such as the median lethal concentration $\left(\mathrm{LC}_{50}\right)$, the no observed effect concentration (NOEC); and the organ (heart, liver, hemoglobin synthesis, etc.) specific toxicity of AEO-7, which are warranted for classifying the toxicity of any chemical compound. Therefore, a comprehensive characterization of the potential toxicity of this surfactant using an aquatic animal model is essential prior to its extensive utilization in various environmental applications. Accordingly, the present study was designed to evaluate the potential toxic effects of AEO-7 using zebrafish embryos as a model for the marine fauna, which is an ideal model system to study environmental toxicity and is widely accepted by the National environmental toxicity and by the National Institutes of Health $(\mathrm{NIH}$, USA) [2, 35-37]. Since no toxicity studies have been performed on AEO-7, we investigated a wide range of concentrations $(0.4,0.8,3.2,6.4,12.8,25, \mu \mathrm{g} / \mathrm{L})$ to assess the $\mathrm{NOEC}$ and the median lethal concentration $\mathrm{LC}_{50}$. In this regard, the selected concentrations were consistent with previously published work using surfactants [34, 38] and within the toxicity rating scale provided by the U.S. Fish and Wildlife Service (USFWS) [39]. In addition, we investigated the potential adverse effects of AEO-7 on the normal embryonic development of zebrafish using organ-specific toxicity assays (cardiotoxicity, neurotoxicity, hepatotoxicity, and hemoglobin synthesis).

\section{Materials and methods Chemicals}

Two compounds were employed as positive controls for general toxicity: diethylaminobenzaldehyde (DEAB, Sigma-Aldrich, Steinheim, Germany), an inhibitor of aldehyde dehydrogenases that causes significant pathologies and mortality in zebrafish embryos [37, 40, 41] and nanoparticles of zinc oxide $(\mathrm{ZnO}$, diameter $<100 \mathrm{~nm}$, catalog \#721077-100G, Sigma-Aldrich, Steinheim, Germany) known to cause mortality and morphological deformities in zebrafish embryos and used previously as a positive control in toxicological studies [42-44]. In addition, in connection with the assays of neurotoxicity, 1-methyl-4-phenyl-1,2,3,6-tetrahydropyridine hydrochloride (MPTP, Sigma-Aldrich, Steinheim, Germany), which causes permanent symptoms of Parkinson's 
disease in these same embryos, was used as the positive control [45].

To facilitate visualization under the microscope, the zebrafish embryos were incubated in E3 egg water (Sigma-Aldrich, Steinheim, Germany) containing N-phenylthiourea (PTU, Sigma-Aldrich, Steinheim, Germany) which inhibits pigmentation with melanin. The AEO-7 surfactant was obtained from Shanghai Dejun Technology Co., Ltd, China. The chemical structure of the surfactant is shown in Additional file 1, Fig. S1. A stock solution of AEO-7 $(2 \mathrm{mg} / \mathrm{L})$ was prepared by adding 5 $\mu \mathrm{L}$ of the viscous liquid to $4.995 \mathrm{~mL}$ PTU medium and overtaxing until fully dissolved. Stock solutions of DEAB, $\mathrm{ZnO}, \mathrm{PTU}$, E3 egg water, and phosphate buffer saline (PBS) were prepared as described previously $[2,37]$.

\section{Zebrafish embryo culture}

The three types of zebrafish (Danio rerio) embryos used were the wild-type $A B$ strain, the naturally transparent Casper strain and $\operatorname{Tg}[$ fabp10: RFP] transgenic $A B$ zebrafish, which express red florescent protein (RFP) in all liver cells [46]. The wild-type animals were maintained in an aquatic system at the Biomedical Research Center (BRC) of Qatar University (QU) and embryos generated by natural pairwise mating, as described in the Zebrafish Book [47]. Dead and unfertilized eggs were removed $4 \mathrm{~h}$ post-fertilization (hpf). Prior to 7 days post-fertilization (dpf), the embryos receive their nourishment from the yolk sac and, thus, no feeding is required [48].

Since different organs of the zebrafish become fully functional at different stages [40], acute toxicity, cardiotoxicity, and hematopoiesis were assessed once every $24 \mathrm{~h}$ for 3 days after initiating exposure at $24 \mathrm{hpf}$, and central nervous system (CNS) toxicity and hepatotoxicity following treatment from 96 to $120 \mathrm{hpf}$, as illustrated in Fig. 1 [36, 37, 40, 41, 49-52]. During early embryogenesis the protective chorion envelope around the embryos might interfere with uptake of the compounds being tested. Therefore, at $24 \mathrm{hpf}$ the zebrafish embryos were dechorionated using a solution of pronase (0.5 mg/mL, Sigma-Aldrich, Steinheim, Germany) [2, 40].

All procedures were conducted in compliance with the guidelines provided by Qatar University and the Department of Research at the Ministry of Public Health, Qatar.

\section{Acute toxicity}

The acute toxicity of AEO-7 was assessed with assay adapted from the guidelines for testing chemical toxicity formulated by the Organization for Economic Co-operation and Development (OECD) (No. 203, 210 and 236) $[3,53,54]$. Since we could find no previous reports on the toxicity of AEO-7 in the scientific literature, a wide range of concentrations was evaluated.

At $24 \mathrm{hpf}, 20$ healthy, dechorionated wild-type embryos were placed into each well of 12-well plates together with $3 \mathrm{~mL}$ of either E3 medium (NC) alone or this same medium with one of six different concentrations $(0.4,0.8,3.2,6.4,12.8$, and $25 \mu \mathrm{g} / \mathrm{L})$ of AEO-7 or $1 \mu \mathrm{M}$ DEAB. Thereafter, cumulative survival and morphological deformities were assessed under a standard stereomicroscope at 96 hpf. Embryos where the fertilized egg had coagulated, no somites formed, no heartbeat was detectable and/or the tail-bud had not detached from the yolk

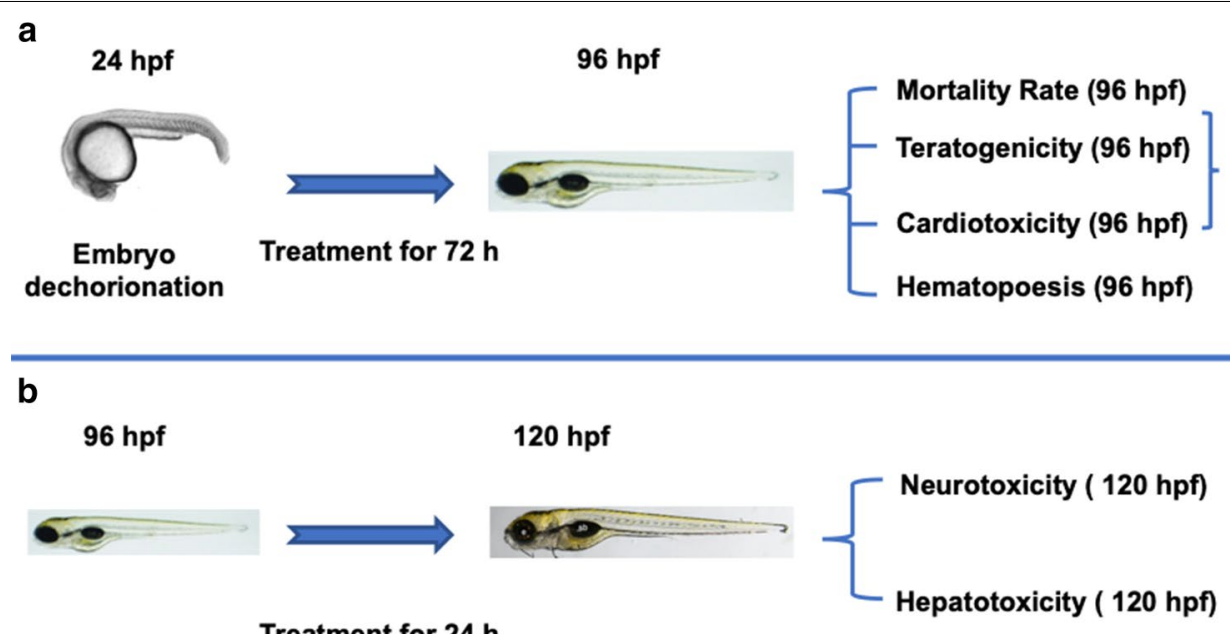

Treatment for $\mathbf{2 4} \mathrm{h}$

Fig. 1 A summary of the experimental procedures utilized to assess different types of toxicity. a At 24 hpf, the embryos were dechorionated and exposed to different concentrations of AEO-7, positive control (PC), and negative control (NC) until 96 hpf, when acute toxicity was assessed. b Starting at $96 \mathrm{hpf}$, the embryos were exposed to different concentrations of AEO-7, PC, and NC for $24 \mathrm{~h}$ and organ toxicity then evaluated 
sac were considered dead. Defects or variations in body length or the size of the eyes, heart or yolk were considered teratogenic effects.

The median lethal dose $\left(\mathrm{LC}_{50}\right)$ with a $95 \%$ confidence interval was calculated by fitting a sigmoidal curve to the data on mortality using the GraphPad Prism 8 software (version 8.2.1, San Diego, CA, USA), as described elsewhere [36, 37, 52]. Variations in body length and the size of the eyes and yolk sac size were captured at 21-fold magnification with the HCImage software and then assessed with the ImageJ software (version 1.52a, NIH, Washington DC, USA) in combination with Java 1.8.0_172 [37, 55].

Both the data on mortality and teratogenicity were utilized to calculate the no observed effect concentration (NOEC) of AEO-7, i.e., the highest concentration that does not cause a significant $(p<0.05)$ effect relative to the negative control (PTU-E3 medium). If the cumulative mortality in the negative control was $>20 \%$, the experiment was repeated. As indicated above, $n=20$ in all cases.

\section{Hatching rate}

At 4-5 hpf, exposure of the embryos in the same manner as described above was initiated and hatching monitored once every $24 \mathrm{~h}$ for 4 days thereafter. The hatching rate was calculated as follows:

$$
\text { Hatching rate }=\frac{\text { number of hatched embryos }}{\text { total number of living embryos }} \times 100 \text {. }
$$

\section{Cardiotoxicity}

For assaying cardiotoxicity, the average peak blood flow, as well as pulse (based on the flow of red blood cells $(\mathrm{RBC})$ ) were monitored in the two major vessels in the trunk of the embryos, the dorsal aorta (DA) and posterior cardinal vein (PCV), as shown in Fig. 4a. RBC tracking was accomplished by algorithms in the video analysis program MicroZebraLab blood flow (version 3.4.4, Viewpoint, Lyon, France). As described above, treatment with $20 \mu \mathrm{g} / \mathrm{L} \mathrm{ZnO}$ was used as the positive control for cardiotoxicity [2, 42]. At $96 \mathrm{hpf}, 10$ embryos exposed to each treatment (Sect. 2.3) were selected at random, anesthetized by immersion in $0.7 \mu \mathrm{M}$ tricaine methane sulfonate (A4050, Sigma-Aldrich, St. Louis, MO, USA) in E3 medium, and imaged as described previously $[2,49,52]$.

\section{Staining for hemoglobin}

To evaluate the effect of AEO-7 on hemoglobin synthesis, Casper embryos were stained with $o$-dianisidine stain (Catalog \#D9143-5G, Sigma, USA) in accordance with a protocol described previously [56]. This compound oxidizes hemoglobin, producing a dark red stain in cells that contain this protein. At $24 \mathrm{hpf}$, healthy embryos were transferred to a 12-well plate and incubated for $96 \mathrm{~h}$ at $28^{\circ} \mathrm{C}$ with PTU (negative control), $1 \mu \mathrm{M} \mathrm{DEAB}$ (positive control), or 0.8 or $3.2 \mu \mathrm{g} / \mathrm{L}$ AEO-7.

In addition, hemoglobin in embryos treated at $96 \mathrm{hpf}$ as described above in Sect. 2.3 was determined with $o$-dianisidine (Sigma-Aldrich, Steinheim, Germany) in accordance with protocols described previously by Paffett-Lugassy and Zon [2, 50, 57]. In brief, the embryos were stained with $o$-dianisidine in the dark for $30 \mathrm{~min}$ as described elsewhere [2], positioned horizontally on microscope slides and embedded in $3.0 \%(\mathrm{w} / \mathrm{v})$ methylcellulose for bright field microscopic imaging (Stemi 508 Zeiss) at $50 \times$ in combination with a Zeiss AxioCam ERc $5 \mathrm{~s}$ camera. The average surface area of erythrocytes stained dark red in 10 embryos in each group was determined using the ImageJ software for comparison to the negative control.

\section{Locomotion (neuromuscular toxicity)}

To assay locomotion, embryos were collected in a Petri dish containing E3 medium, abnormal and unfertilized embryos discarded, and healthy embryos incubated at $28.5^{\circ} \mathrm{C}$. At $96 \mathrm{hpf}, 15$ embryos were transferred to each well of a 12-well plate and incubated for $24 \mathrm{~h}$ at $28{ }^{\circ} \mathrm{C}$ with E3 medium (negative control), $100 \mu \mathrm{M}$ MPTP (positive control), or (iii) 0.8 or $3.2 \mu \mathrm{g} / \mathrm{L}$ AEO-7. Thereafter, each embryo was placed separately in a well on a 96-well plate for evaluation of locomotion utilizing the ViewPoint ZebraBox technology (ViewPoint Life Sciences Lyon, France) as described previously $[41,58]$.

In brief, the 96-well plates were placed in a chamber at $28.5{ }^{\circ} \mathrm{C}$ and irradiated for $20 \mathrm{~min}$ with white light to allow the embryos to adapt to this environment. Then, the movement of the embryos was measured under the following conditions: an initial 10-min period of darkness accompanied by two repeated bright light cycles for $10 \mathrm{~min}$, which was separated by $10 \mathrm{~min}$ of darkness. The neurotoxicity was determined through measurement of the average total distance moved after a cycle of $60 \mathrm{~min}$ and by assessing the response of the embryos by the dark-light cycles. The results were compared to the negative and positive controls.

\section{Hepatotoxicity}

Hepatotoxicity was assessed in $\mathrm{Tg}$ [fabp10: RFP] transgenic $A B$ zebrafish, which express red fluorescent protein (RFP) in their hepatocytes, allowing good-quality staining of the liver. At $96 \mathrm{hpf}$, the embryos were incubated for $24 \mathrm{~h}$ at $28{ }^{\circ} \mathrm{C}$ with E3 medium (negative control), $1 \%$ ethanol (positive control) or 0.8 or $3.2 \mu \mathrm{g} / \mathrm{L}$ AEO-7, following which liver size (as an indication of necrosis and hepatomegaly) and yolk retention (as a 
reflection of hepatic lipid metabolism) were evaluated as described previously [40, 41]. At $120 \mathrm{hpf}$, the liver of zebrafish embryos is fully developed [59].

For determination of liver size, this fluorescent organ was examined in 10 embryos exposed to each treatment with a fluorescence stereomicroscope (Olympus MVX10) and a digital camera (Olympus DP71). The images were filtered with RFP and liver size analyzed utilizing the DanioScope software (Noldus, Wageningen, Netherlands) [40].

Yolk retention was assessed by treatment with Oil Red O (ORO) (Catalog \#1320-06-5, Sigma-Aldrich, USA), a lysochrome, fat-soluble stain for neutral triglycerides and lipids, as described by Yoganantharjah and his colleagues (2017) [60]. In brief, $0.035 \mathrm{~g}$ ORO powder was added to $7 \mathrm{~mL} 100 \%$ isopropanol and dissolving by stirring overnight with a magnetic stirrer at room temperature. To obtain the staining solution utilized, an aliquot of this stock solution was mixed with an equal volume of $10 \%$ isopropanol in Milli-Q water.

Following treatment as described above, the PTUE3 medium was removed from the embryos by washing with $60 \%$ isopropanol and they were then placed in $1 \mathrm{~mL}$ of the staining solution for $75 \mathrm{~min}$. Thereafter, the embryos were washed for $30 \mathrm{~s}$ with $60 \%$ isopropanol and then rinsed again for $3 \mathrm{~min}$ in $60 \%$ isopropanol, followed by a 30 -s wash in $1 \%$ PBS. Next, the ORO stain was extracted from the embryos for quantification.

For this purpose, 5 embryos were pooled in an Eppendorf tube, with 5 or 6 such tubes for each treatment. Following removal of the PBS, $250 \mathrm{~mL} 4 \%$ ethanol in isopropanol was added to each tube and the samples vortexed briefly and then incubated overnight at room temperature to ensure complete extraction of the ORO stain. Finally, $200 \mathrm{~mL}$ of the solution was pipetted into each well of a 96-well plate and the OD (absorbance) at $495 \mathrm{~nm}$ determined with a Tecan GENios Pro 200 spectrophotometer.

\section{Statistical analysis}

In most cases, the values for the treated and negative control were compared statistically with one-way ANOVA followed by the Dunnett test and paired twotailed Student's $t$ test. In the case of the hatching assay, the Chi-square test was utilized for this purpose. Statistical significance is indicated as ${ }^{*} p<0.05 ;{ }^{* * *} p<0.01$; or $* * * 0<001$. All statistical analyses were performed with the GraphPad Prism 8 software (version 8.2.1).

\section{Results and discussion}

AEO-7 is extremely toxic towards zebrafish embryos

Zebrafish embryos are most sensitive to xenobiotic from 24 to 96 hpf [40, 61-63]. At 96 hpf, mortality following exposure to $100 \mu \mathrm{M} \mathrm{DEAB}$ (the positive control) was $100 \%$, with severe teratogenicity, including abnormalities in the heart and yolk sac edema (Fig. 2a). The $\mathrm{LC}_{50}$ calculated for DEAB was $24.1 \mu \mathrm{M}$ (Fig. 2c).

In the case of exposure to AEO-7, no significant mortality was observed at $96 \mathrm{hpf}$ with $0.4-12.8 \mu \mathrm{g} / \mathrm{L}$, whereas mortality was $100 \%$ at $25 \mu \mathrm{g} / \mathrm{L}$ (Fig. $2 \mathrm{~b}$ ), with an $\mathrm{LC}_{50}$ of $18.3 \mu \mathrm{g} / \mathrm{L}$ (Fig. 2c). Thus, according to the Fish and Wildlife Service Acute Toxicity Rating Scale [39], the AEO-7 surfactant would be classified as "super toxic".

Moreover, $6.4 \mu \mathrm{g} / \mathrm{L} \mathrm{AEO-7}$ reduced the size of the embryo's eyes (Fig. 2f). At the same time, this surfactant decreased body length and increased the size of the yolk sac only at a concentration of $12.8 \mu \mathrm{g} / \mathrm{L}$ (Fig. $2 \mathrm{~d}$, e), which caused a wide range of other embryopathies as well.

\section{AEO-7 did not affect the hatching rate (HR)}

The rate of hatching, which normally occurs with zebrafish embryo from 48 to $96 \mathrm{hpf}$, is a critical indicator of the developmental state of these embryos [64,65]. At a concentration of $25 \mu \mathrm{g} / \mathrm{L}$, AEO-7 eliminated hatching (Fig. 3). The explanation for the pronounced difference from lower concentrations, which had no impact on this process, is unknown, but it is possible that the chorion protects against lower concentrations of this compound $[66,67]$. The reduction in hatching with higher concentrations of AEO-7 may reflect structural and functional disturbances $[68,69]$ and/or the inability of the embryo to break out of its eggshell due to developmental delay [70].

\section{Exposure to AEO-7 induces cardiac dysfunction in zebrafish embryos}

Zebrafish have proven to be an excellent model for studying the cardiotoxic effects of xenobiotics [41, 51], with an overall success rate for predicting cardiotoxic and non-cardiotoxic drugs of $100 \%$ according to some investigators $[71,72]$ and a rating of excellent $(>85 \%)$ for identifying cardiovascular toxins based on the criteria proposed by the European Center for the Validation of Alternative Methods [72]. The dorsal aorta (DA), the major axial artery in the trunk, is one of the vessels that form first during the early development of all vertebrates. This aorta forms immediately below the notochord and above the posterior cardinal vein (PCV), which is the major axial vein in the zebrafish trunk (Fig. 4a) [73].

At a concentration of $3.2 \mu \mathrm{g} / \mathrm{L}, \mathrm{AEO}-7$ elevated the heart rate of zebrafish embryos (Fig. 4b), which is 


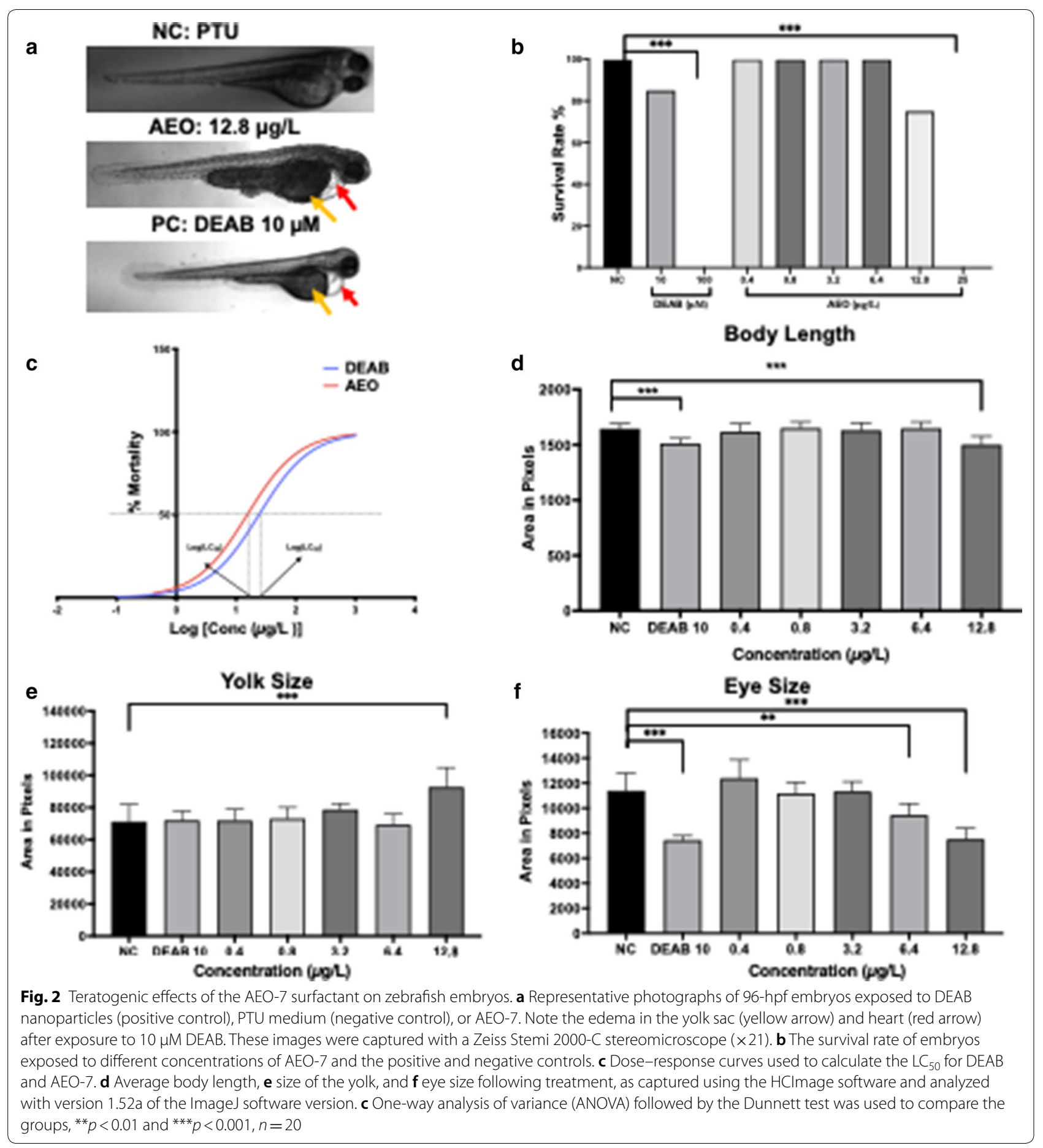

indicative of cardiovascular dysfunction. The NOEC for this effect was calculated to be $0.8 \mu \mathrm{g} / \mathrm{L}$, which is why we used this concentration and the higher concentration of $3.2 \mu \mathrm{g} / \mathrm{L}$ in subsequent toxicity assays. In 


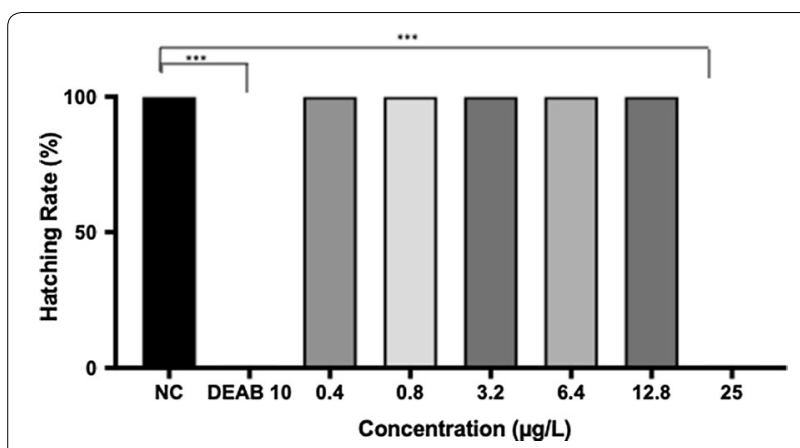

Fig. 3 The effect of AOE-7 on the hatching of zebrafish embryos. The Chi-square test was used for comparison to the negative and positive controls, ${ }^{*} p<0.05 ;{ }^{* *} p<0.01 ;{ }^{* * *} p<0.001, n=20$

agreement with previous reports [74, 75] (Fig. 4b, c), $\mathrm{ZnO}$ showed a significant decrease in the heart rate of the zebrafish embryos as shown in Fig. 4b, c.

\section{AEO-7 alters the locomotion of zebrafish embryos}

Neurotoxic pollutants are an emerging issue that potentially causes serious threats to vertebrate and invertebrate populations in the ecosystems. Despite an increasing number of reports of species showing altered behavior, neurotoxicity assessment for species in the environment is not required and, therefore, mostly not performed. However, considering the increasing numbers of environmental contaminants with neurotoxic potential, we believe that eco-neurotoxicity should also be considered in risk assessment [76]. The behavioral response of the zebrafish embryo is currently seen as a useful endpoint for identifying neurotoxic chemicals [49, 76, 77]. The locomotor response for various neurotoxicants can be evaluated by different type of assays such as locomotion or tail flicking assays [37]. For instance, in the locomotion assay, chemically treated embryos are exposed to alternating light/dark episodes. In most cases, the affected or intoxicated zebrafish embryos exhibit weak or increased movement when they are stimulated by light $[78,79]$.

In our study, we assessed the locomotor response of zebrafish embryos treated with AEO-7. MPTP (PC), which has been identified as a neurotoxin in humans and zebrafish [80], attenuated the total distance that our embryos moved (Fig. 5a, b). In addition, exposure to this compound altered their locomotive behavior. These findings are consistent with the report by Wang and colleagues [26] that AEO-7 reduces the number of periods of rest, as well as the total and waking activity of zebrafish embryos in a concentration-dependent manner. Thus, we confirm here that AEO-7 has a toxic influence on the locomotor activity of zebrafish embryos.

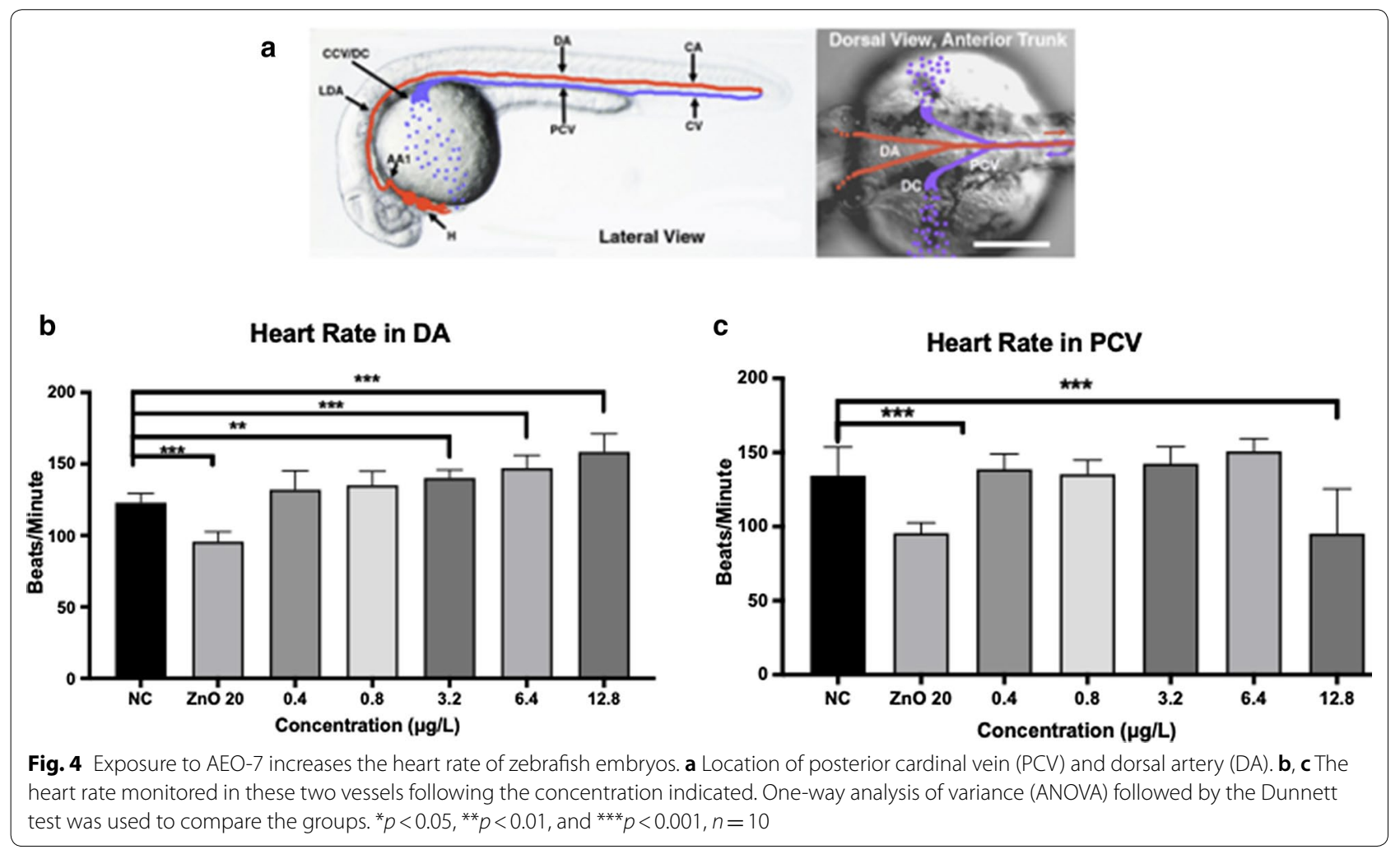




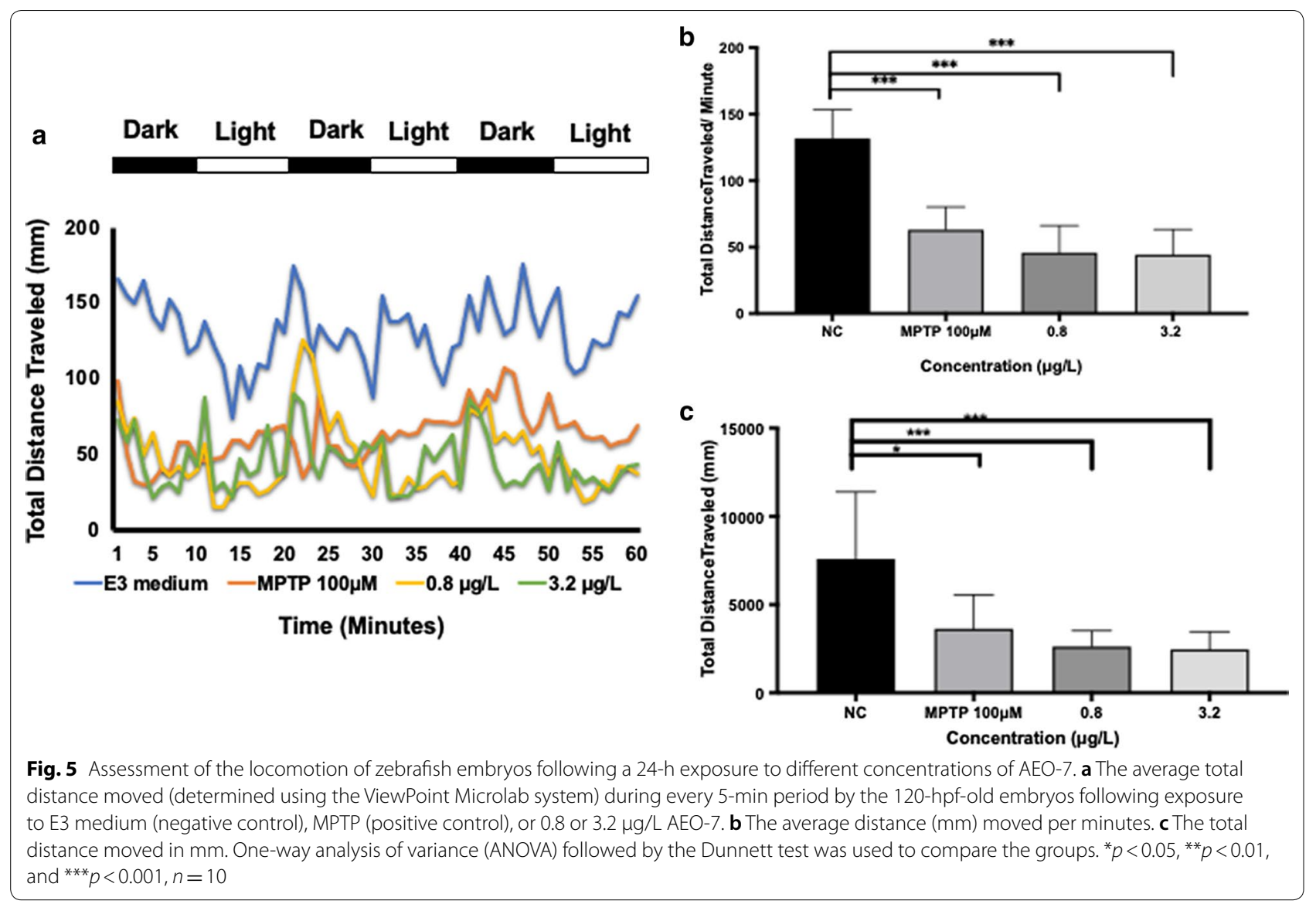

\section{a}
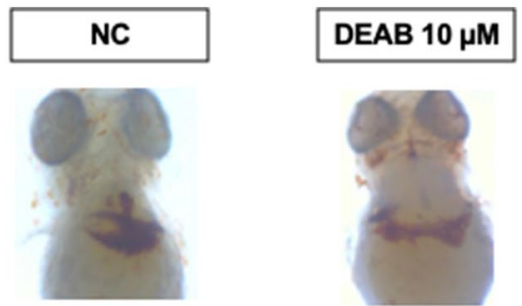

b
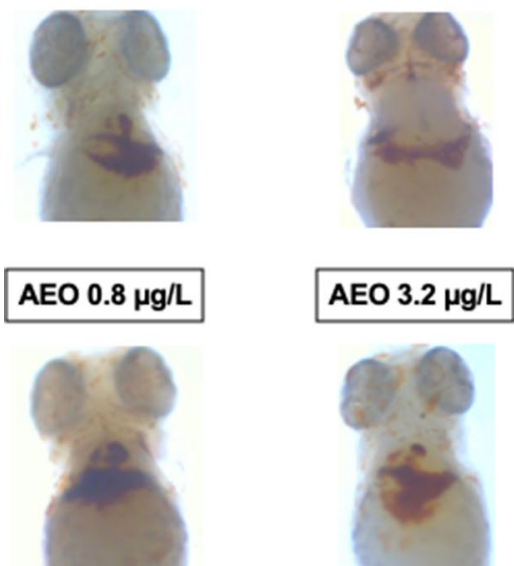

Fig. 6 The influence of AEO-7 on the level of hemoglobin in zebrafish embryos. a Representative images depicting o-dianisidine staining of the yolk sac of 72-hpf zebrafish embryos exposed to the negative control, DEAB, or 0.8 or $3.2 \mu \mathrm{g} / \mathrm{L}$ AEO-7. b The number of erythrocytes stained by o-dianisidine in the embryos described in a. One-way analysis of variance (ANOVA) followed by the Dunnett test was used to compare the groups. ${ }^{*} p<0.05,{ }^{* *} p<0.01$, and ${ }^{* * *} p<0.001, n=10$ 


\section{At its NOEC, AEO-7 does not cause hematopoietic toxicity} in zebrafish embryos

As shown in Fig. 6a, b, at its NOEC (0.8 $\mu \mathrm{g} / \mathrm{L})$, AEO-7 did not affect the level of hemoglobin in our zebrafish embryos, whereas, at a concentration of $3.2 \mu \mathrm{g} / \mathrm{L}$, the number of hemoglobin-positive cells was reduced. This reduction could reflect decreased production of red blood cells in the bone marrow and/or lowered hemoglobin synthesis in erythrocytes due to blockage of heme synthesis $[81,82]$.

\section{At its NOEC, AEO-7 exerts no adverse effect on hepatic function in zebrafish embryos}

Zebrafish have been shown to be a good model for predicting hepatotoxicity [83-85], probably because the enzymes and pathways involved in xenobiotic metabolism (e.g., the aryl hydrocarbon receptor and isozymes of cytochrome P-450 and aldehyde dehydrogenase) are all evolutionarily conserved and all functional from the early stages of the development of zebrafish embryos, including our experimental window $[74,86,87]$.

By $120 \mathrm{hpf}$, zebrafish embryos have normally consumed their entire yolk, which is $70 \%$ lipid, and begins to seek exogenous sources of food [88]. Since the yolk is

\section{a}
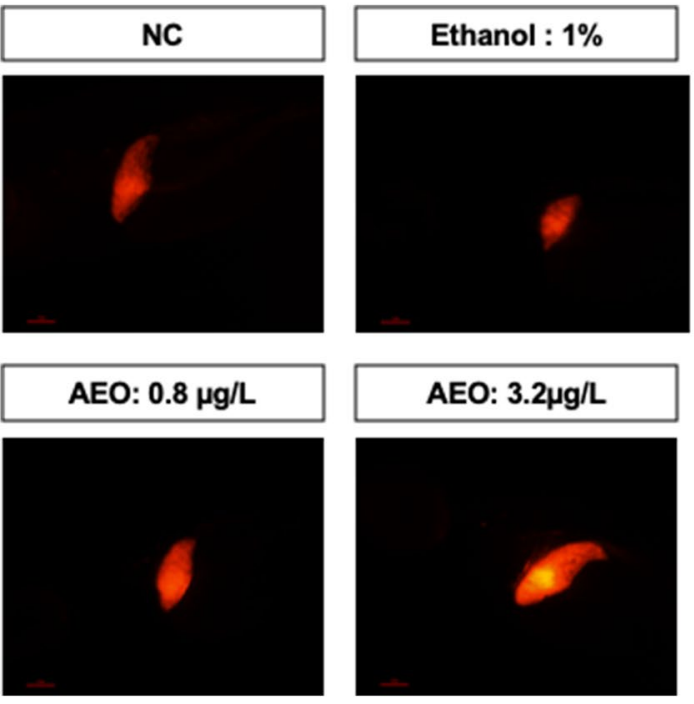

C

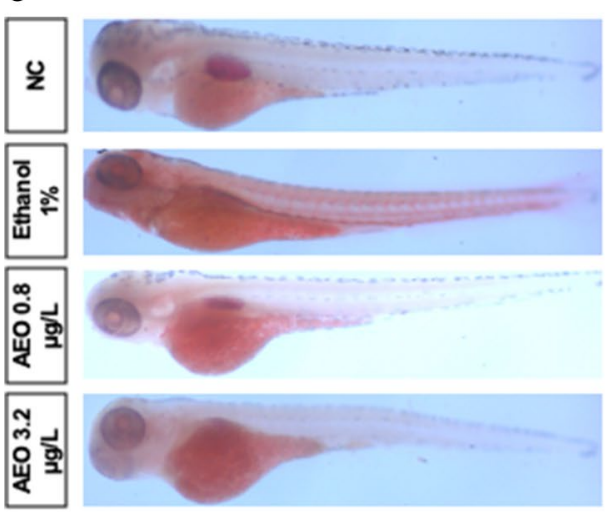

Ethanol : $1 \%$

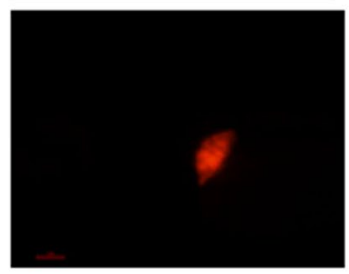

AEO: $3.2 \mu \mathrm{g} / \mathrm{L}$

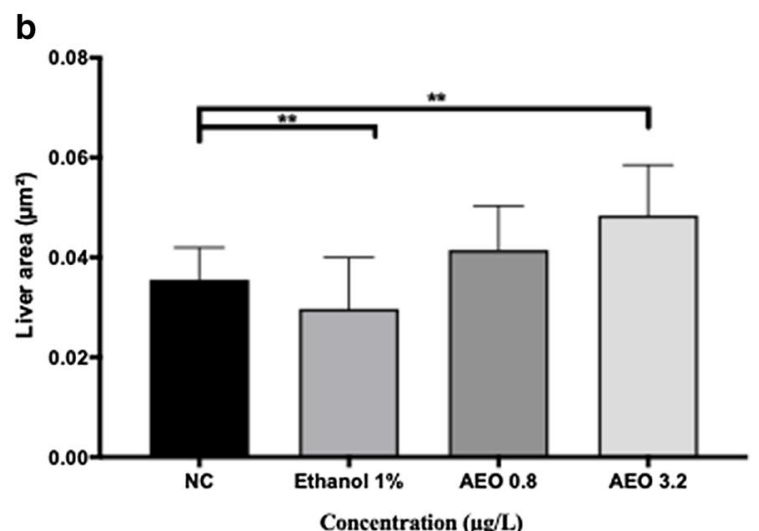

d

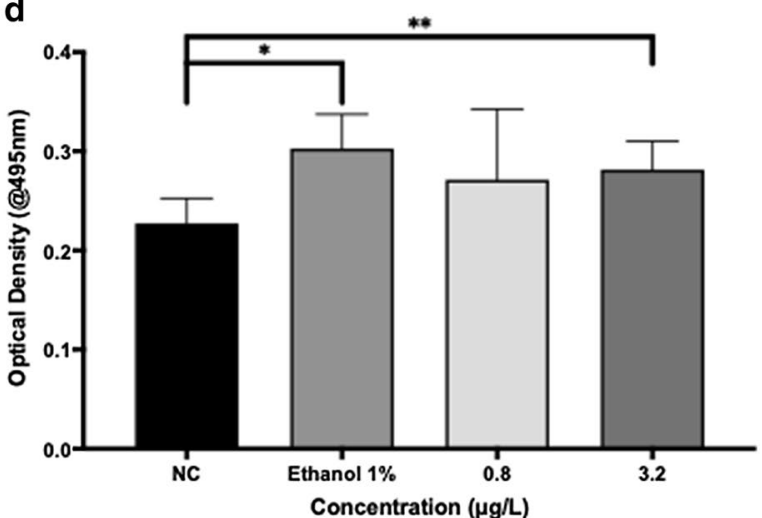

Fig. 7 The effect of AEO-7 on liver size and yolk retention in zebrafish embryos. a Representative images depicting the size of the liver following the different treatments (note the hepatomegaly or necrosis). $\mathbf{b}$ The RFP area of the liver $\left(\mu \mathrm{m}^{2}\right)$ following the different treatments. $\mathbf{c}$ Representative images of yolk retention following the different treatments. $\mathbf{d}$ The levels of neutral triglycerides and total lipids in the entire embryos following the different treatments. Paired two-tailed Student's $t$ test was employed to compare the treated groups with the negative control. ${ }^{*} p<0.05{ }^{* *} p<0.01$, and ${ }^{* * *} p<0.001, n=10$ 
metabolized primarily by the liver [89], retention of the yolk is an indirect indication of impaired liver function [90-92].

Hence, we assessed hepatotoxicity in two different ways, e.g., on the basis of liver size and yolk retention. As expected, $1 \%$ ethanol decreased the size of the liver, indicating necrosis $[41,49,93]$. At its $\operatorname{NOEC}(0.8 \mu \mathrm{g} / \mathrm{L})$, AEO-7 had no influence on liver size, while hepatomegaly was observed at $3.2 \mu \mathrm{g} / \mathrm{L}$ (Fig. 7a, b). Secondly, as shown in Fig. 7, exposure of embryos to $1 \%$ ethanol (positive control) elevated their content of lipid, in agreement with previous studies [41, 49, 93]. At its NOEC, AEO-7 resulted in a minor, but statistically insignificant increase in lipid retention, whereas the higher concentration of this compound enhanced this retention significantly (Fig. 7c, d).

\section{Future prospects}

We are still exploring the toxicity of alternative compounds, whether synthesized or commercially available in the market, to be safely used instead of AEO7. It is worth mentioning that we are currently investigating the toxicity of several commercial surfactants that are widely used in different kinds of applications, such as corrosion inhibitor and cosmetics products. For instance, recently, we investigated in our lab the toxicity of a cationic surfactant, SAPDMA, which we classified as "moderately toxic" based on its $\mathrm{LC}_{50}(2.3 \mathrm{mg} / \mathrm{L})$ [38]. Furthermore, we are planning to study the toxicity of the whole AEO series; including AEO-3, AEO-4, AEO-5, AEO-9, and AEO-15 to find "a green" alternative for AEO-7.

\section{Conclusions}

The results of all of the toxicity assays-including calculation of the $\mathrm{LC}_{50}$ and NOEC values, as well as evaluation of potential toxic effects on the heart, hematopoiesis, locomotion and the liver-indicate strongly that at least at the concentrations tested the non-ionic surfactant AEO-7 exerts potent concentration-dependent toxic effects on organ development in zebrafish embryos. To our knowledge, our investigation is the first complete assessment of the toxicity of AEO-7 surfactant towards a model freshwater organism.

Indeed, the mortality of 96-hpf embryos following exposure to AEO-7 rose with increasing concentration, becoming $100 \%$ at $25 \mu \mathrm{g} / \mathrm{L}$. The $\mathrm{LC}_{50}$ and NOEC values were 15.35 and $0.8 \mu \mathrm{g} / \mathrm{L}$, respectively. On the basis of these values alone, we conclude that according to the USFWS Acute Toxicity Rating Scale (Additional file 1, Table S1) [39], AEO-7 should be classified as "super toxic". Therefore, industrial use of AEO-7 and its presence in the aquatic environment should be re-evaluated and monitored carefully by different environmental and public health organizations.

\section{Supplementary information}

Supplementary information accompanies this paper at https://doi. org/10.1186/s12302-020-00429-z.

Additional file 1: Figure S1. The chemical structure of the AEO7 molecule [1]. Table S1. Acute Toxicity Rating Scale by the U.S. Fish and Wildlife Service (USFWS) (El-Harbawi 2014).

\section{Acknowledgments}

We would like to thank the following undergraduate students for their technical helps in the experiments: Bana Salim Alsahan and Asma Jumah S L Al-Mesaifri.

\section{Authors' contributions}

MA writing, data analysis, visualization, provision of resources, project administration, acquisition of funding. GP writing — review and editing, acquisition of funding. AFM visualization, validation, writing. NY development of methodology, use of software, formal analysis, performance of experiments, writing, visualization. SID use of software, formal analysis, validation. AMA supervision, performance of experiments. ABR development of methodology, performance of experiments. MHS development of methodology, performance of experiments. HE-C development of methodology, performance of experiments. GKN conceptualization, validation, formal analysis, provision of resources, storage of data, visualization, supervision, project administration, acquisition of funding. All authors read and approved the final manuscript.

\section{Funding}

This work was supported by an internal grant (QUCP-CHS-2019-1) awarded by Qatar University to MA, as well as by an international research collaboration co-fund grant (IRCC-2019-007) by this same university to GKN and GFP. In addition, this work was made possible by a grant (UREP26-097-3-040) from the Qatar National Research Fund (a member of the Qatar Foundation). The statements made herein are solely the responsibility of the authors.

Availability of data and materials Not applicable.

Ethics approval and consent to participate Not applicable.

\section{Consent for publication}

Not applicable.

\section{Competing interests}

None of the authors has any competing interests to declare.

\section{Author details}

${ }^{1}$ Biomedical Research Center, QU Health, Qatar University, P.O. Box 2713, Doha, Qatar. ${ }^{2}$ Department of Biomedical Sciences, College of Health Sciences, QU Health, Qatar University, Women's Science building, C01, P.O. Box 2713, Doha, Qatar. ${ }^{3}$ Department of Biology, Chemistry, and Environmental Sciences, College of Arts and Sciences, American University of Sharjah, P.O. Box 26666, Sharjah, United Arab Emirates. ${ }^{4}$ Center for Advanced Materials, Qatar University, P.O. Box 2713, Doha, Qatar. ${ }^{5}$ Department of Chemical Engineering, College of Engineering, Qatar University, P.O. Box 2713, Doha, Qatar. ${ }^{6}$ Department of Human Genetics, Sidra Medicine, P.O. Box 26999, Doha, Qatar. ${ }^{7}$ Department of Medical Laboratory Sciences, University of Sharjah, P.O. Box 27272, Sharjah, United Arab Emirates.

Received: 16 September 2020 Accepted: 30 October 2020 Published online: 11 November 2020 


\section{References}

1. Bahgat Radwan A et al (2017) Corrosion inhibition of API X120 steel in a highly aggressive medium using stearamidopropyl dimethylamine. J Mol Liq 236:220-231

2. Al-Kandari $\mathrm{H}$ et al (2019) Ecotoxicological assessment of thermally- and hydrogen-reduced graphene oxide/ $/ \mathrm{TiO}_{2}$ photocatalytic nanocomposites using the zebrafish embryo model. Nanomaterials (Basel, Switzerland) 9(4):488

3. OECD, Test No. 236: fish embryo acute toxicity (FET) Test. 2013.

4. Radwan AB et al (2017) Corrosion inhibition of API X120 steel in a highly aggressive medium using stearamidopropyl dimethylamine. J Mol Liq 236:220-231

5. Darling D, Rakshpal R (1998) Green chemistry applied to corrosion and scale inhibitors. NACE International, Houston

6. Analysis, G.S.M.t.I.T. The future of surfactants to 2022. 2017. https://www. smithers.com/services/market-reports/materials/the-future-of-surfactant s-to-2022.

7. Gudiña EJ et al (2016) Valorization of agro-industrial wastes towards the production of rhamnolipids. Bioresour Technol 212:144-150

8. Analysis CR. Assessing the sustainability and performance of green surfactants. 2020. https://ihsmarkit.com/research-analysis/assessing-susta inability-and-performance-of-green-surfactants.html.

9. Belanger S et al (2006) Special issue on the environmental risk assessment of alcohol ethoxylate nonionic surfactant. Ecotoxicol Environ Saf 64:1-2

10. Olkowska E, Ruman M, Polkowska Z (2014) Occurrence of surface active agents in the environment. J Anal Methods Chem 2014:769708

11. Cvetkovic Z, Vidaković-Cifrek Z, Puntarić D (2006) Toxicity of surfactants to green microalgae Pseudokirchneriella subcapitata and Scenedesmus subspicatus and to marine diatoms Phaeodactylum tricornutum and Skeletonema costatum. Chemosphere 61:1061-1068

12. Jin D et al (2007a) Effects of concentration, head group, and structure of surfactants on the degradation of phenanthrene. J Hazard Mater 144(1-2):215-221

13. Boeije GM et al (2006) Ecotoxicity quantitative structure-activity relationships for alcohol ethoxylate mixtures based on substance-specific toxicity predictions. Ecotoxicol Environ Saf 64(1):75-84

14. Belanger SE et al (2000) Responses of aquatic communities to 25-6 alcohol ethoxylate in model stream ecosystems. Aquat Toxicol 48(2):135-150

15. Jin D et al (2007b) Effects of concentration, head group, and structure of surfactants on the degradation of phenanthrene. J Hazard Mater 144(1):215-221

16. Wildish DJ (1972) Acute toxicity of polyoxyethylene esters and polyoxyethylene ethers to S. Salar and G. Oceanicus. Water Res 6(7):759-762

17. Vaughan $M$, van Egmond $R$ (2010) The use of the zebrafish (Danio rerio) embryo for the acute toxicity testing of surfactants, as a possible alternative to the acute fish test. Altern Lab Anim 38(3):231-238

18. El-Lateef H (2014) Anti-corrosive activities of some novel surfactants based on vegetable oils. Eur Chem Bull 3:437-440

19. Zhu Y et al (2017) A review of surfactants as corrosion inhibitors and associated modeling. Prog Mater Sci 90:159-223

20. Malik M et al (2011) Anti-corrosion ability of surfactants: a review. Int J Electrochem Sci 6:1927-1948

21. Sliem MH et al (2019) AEO7 surfactant as an eco-friendly corrosion inhibitor for carbon steel in HCl solution. Sci Rep 9(1):2319-2319

22. Hegazy MA et al (2016) Novel cationic surfactants for corrosion inhibition of carbon steel pipelines in oil and gas wells applications. J Mol Liq 214:347-356

23. Zhu Y, Free ML, Cho J-H (2016) Integrated evaluation of mixed surfactant distribution in water-oil-steel pipe environments and associated corrosion inhibition efficiency. Corros Sci 110:213-227

24. Heakal FE-T, Elkholy AE (2017) Gemini surfactants as corrosion inhibitors for carbon steel. J Mol Liq 230:395-407

25. Rhein L. Surfactant action on skin and hair. Cleansing and skin reactivity mechanisms. In: Handbook for cleaning/decontamination of surfaces, 2007. p. 305-69

26. Wang $Y$ et al (2015a) Exploring the effects of different types of surfactants on zebrafish embryos and larvae. Sci Rep 5(1):10107

27. Cserháti T (1995) Alkyl ethoxylated and alkylphenol ethoxylated nonionic surfactants: interaction with bioactive compounds and biological effects. Environ Health Perspect 103(4):358-364
28. Jardak K, Drogui P, Daghrir R (2016) Surfactants in aquatic and terrestrial environment: occurrence, behavior, and treatment processes. Environ Sci Pollut Res 23(4):3195-3216

29. Yuan C et al (2014) The determination methods for non-ionic surfactants. I Chem Pharm Res 6:2238-2242

30. CN105886137A. High-density low temperature quickly dissolved washing powder and preparation method thereof. 2016. https://patents.googl e.com/patent/CN105886137A/en.

31. CN1152127C. External surface cleaning agent of airplane body. 2004

32. CN103695197A. High-concentration laundry detergent. 2014.

33. CN101200676A. Non-toxic class floor furniture detergent having sterilizing function and preparation method thereof. 2009. https://paten ts.google.com/patent/CN101200676A/en.

34. Wang Y et al (2015b) Exploring the effects of different types of surfactants on zebrafish embryos and larvae. Sci Rep 5:10107-10107

35. Meyers JR (2018) Zebrafish: development of a vertebrate model organism. Curr Protoc Essent Lab Tech 16(1):e19

36. Nasrallah $\mathrm{G}$ et al (2018) Ecotoxicological assessment of $\mathrm{Ti}_{3} \mathrm{C}_{2} \mathrm{~T}_{\mathrm{x}}$ (MXene) using zebrafish embryo model. Environ Sci Nano 5(4):1002-1011

37. Younes $\mathrm{N}$ et al (2018a) Toxicity evaluation of selected ionic liquid compounds on embryonic development of Zebrafish. Ecotoxicol Environ Saf 161:17-24

38. Al-Jamal O et al (2020) Organ-specific toxicity evaluation of stearamidopropyl dimethylamine (SAPDMA) surfactant using zebrafish embryos. Sci Total Environ 741:140450

39. El-Harbawi M (2014) Toxicity measurement of imidazolium ionic liquids using acute toxicity test. Proc Chem 9:40-52

40. Cornet $C$ et al (2017) ZeGlobalTox: an innovative approach to address organ drug toxicity using zebrafish. Int J Mol Sci 18(4):864

41. Abou-Saleh H et al (2019) Impaired liver size and compromised neurobehavioral activity are elicited by chitosan nanoparticles in the zebrafish embryo model. Nanomaterials (Basel, Switzerland) 9(1):122

42. Choi JS et al (2016) developmental toxicity of zinc oxide nanoparticles to zebrafish (Danio rerio): a transcriptomic analysis. PLoS ONE $11(8): e 0160763$

43. Kteeba S et al (2017) Zinc oxide nanoparticle toxicity in embryonic zebrafish: mitigation with different natural organic matter. Environ Pollut (Barking, Essex: 1987) 230:1125-1140

44. Bai W et al (2010) Toxicity of zinc oxide nanoparticles to zebrafish embryo: a physicochemical study of toxicity mechanism. J Nanopart Res 12:1645-1654

45. Wen L et al (2008) Visualization of monoaminergic neurons and neurotoxicity of MPTP in live transgenic zebrafish. Dev Biol 314(1):84-92

46. Dong PD et al (2007) Fgf10 regulates hepatopancreatic ductal system patterning and differentiation. Nat Genet 39(3):397-402

47. Monte W, A guide for the laboratory use of zebrafish Danio rerio. In: The zebrafish book. 1993

48. Clift D et al (2014) High-throughput analysis of behavior in zebrafish larvae: effects of feeding. Zebrafish 11(5):455-461

49. Younes N et al (2020) "Safe" chitosan/zinc oxide nanocomposite has minimal organ-specific toxicity in early stages of zebrafish development. ACS Biomater Sci Eng 6(1):38-47

50. Fernández-Murray JP et al (2016) Glycine and folate ameliorate models of congenital sideroblastic anemia. PLoS Genet 12(1):e1005783

51. Zakaria ZZ et al (2018) Using zebrafish for investigating the molecular mechanisms of drug-induced cardiotoxicity. Biomed Res Int 2018:1642684

52. Rasool K et al (2018) "Green" ZnO-interlinked chitosan nanoparticles for the efficient inhibition of sulfate-reducing bacteria in inject seawater. ACS Sustain Chem Eng 6(3):3896-3906

53. OECD. Test No. 203: fish, acute toxicity test. 2019

54. OECD. Test No. 210: fish, early-life stage toxicity test. 2013.

55. Lemieux $S$ et al (1999) Comparison of two techniques for measurement of visceral adipose tissue cross-sectional areas by computed tomography. Am J Hum Biol 11(1):61-68

56. Leet JK et al (2014) High-content screening in zebrafish embryos identifies butafenacil as a potent inducer of anemia. PLoS ONE 9(8):e104190-e104190

57. Paffett-Lugassy NN, Zon LI (2005) Analysis of hematopoietic development in the zebrafish. Methods Mol Med 105:171-198 
58. Younes $\mathrm{N}$ et al (2018b) Toxicity evaluation of selected ionic liquid compounds on embryonic development of Zebrafish. Ecotoxicol Environ Saf. 161:17-24

59. Chu J, Sadler KC (2009a) New school in liver development: lessons from zebrafish. Hepatology (Baltimore, MD) 50(5):1656-1663

60. Yoganantharjah P et al (2017) Rapid quantification of neutral lipids and triglycerides during zebrafish embryogenesis. Int J Dev Biol 61(1-2):105-111

61. Yan $\mathrm{H}$ et al (2012) Functional mesoporous silica nanoparticles for photothermal-controlled drug delivery in vivo. Angew Chem Int Ed 51(33):8373-8377

62. Wheeler JR, Maynard SK, Crane M (2014) An evaluation of fish early life stage tests for predicting reproductive and longer-term toxicity from plant protection product active substances. Environ Toxicol Chem 33(8):1874-1878

63. Hallare AV, Köhler HR, Triebskorn R (2004) Developmental toxicity and stress protein responses in zebrafish embryos after exposure to diclofenac and its solvent. DMSO Chemosphere 56(7):659-666

64. Korenbrot Jl et al (2013) EML1 (CNG-modulin) controls light sensitivity in darkness and under continuous illumination in zebrafish retinal cone photoreceptors. J Neurosci 33(45):17763-17776

65. Li J et al (2018) Xiaoaiping induces developmental toxicity in zebrafish embryos through activation of ER stress, apoptosis and the Wnt pathway. Front Pharmacol 9:1250-1250

66. Gellert G, Heinrichsdorff J (2001) Effect of age on the susceptibility of zebrafish eggs to industrial wastewater. Water Res 35(15):3754-3757

67. Stones DH et al (2017) Zebrafish (Danio rerio) as a vertebrate model host to study colonization, pathogenesis, and transmission of foodborne Escherichia coli 0157. mSphere 2(5):e00365-17

68. Samaee SM et al (2015) Efficacy of the hatching event in assessing the embryo toxicity of the nano-sized $\mathrm{TiO}_{2}$ particles in zebrafish: a comparison between two different classes of hatching-derived variables. Ecotoxicol Environ Saf 116:121-128

69. Liu L et al (2016) Relative developmental toxicity of short-chain chlorinated paraffins in Zebrafish (Danio rerio) embryos. Environ Pollut 219:1122-1130

70. Sinha P, Kanamadi RD (2000) Effect of mercurial fungicide Emisan ${ }^{\circledR}-6$ on the embryonic developmental stages of zebrafish, Brachydanio (Danio) rerio. J Adv Zool 21:12-18

71. Burns CG et al (2005) High-throughput assay for small molecules that modulate zebrafish embryonic heart rate. Nat Chem Biol 1(5):263-264

72. Ducharme NA et al (2015) Comparison of toxicity values across zebrafish early life stages and mammalian studies: implications for chemical testing. Reprod Toxicol 55:3-10

73. Geudens I et al (2019) Artery-vein specification in the zebrafish trunk is pre-patterned by heterogeneous Notch activity and balanced by flow-mediated fine-tuning. Development (Cambridge, England) 146(16):dev181024

74. Du J et al (2016) Developmental toxicity and DNA damage to zebrafish induced by perfluorooctane sulfonate in the presence of $\mathrm{ZnO}$ nanoparticles. Environ Toxicol 31(3):360-371

75. Bessemer RA et al (2015) Cardiorespiratory toxicity of environmentally relevant zinc oxide nanoparticles in the freshwater fish Catostomus commersonii. Nanotoxicology 9(7):861-870
76. Legradi JB et al (2018) An ecotoxicological view on neurotoxicity assessment. Environ Sci Eur 30(1):46

77. Ogungbemi A et al (2019) Hypo- or hyperactivity of zebrafish embryos provoked by neuroactive substances: a review on how experimental parameters impact the predictability of behavior changes. Environ Sci Eur $31(1): 88$

78. Irons TD et al (2010) Acute neuroactive drug exposures alter locomotor activity in larval zebrafish. Neurotoxicol Teratol 32(1):84-90

79. Selderslaghs IWT et al (2010) Locomotor activity in zebrafish embryos: a new method to assess developmental neurotoxicity. Neurotoxicol Teratol 32(4):460-471

80. Anichtchik OV et al (2004) Neurochemical and behavioural changes in zebrafish Danio rerio after systemic administration of 6-hydroxydopamine and 1-methyl-4-phenyl-1,2,3,6-tetrahydropyridine. J Neurochem 88(2):443-453

81. Souza PC, Bonilla-Rodriguez GO (2007) Fish hemoglobins. Brazil J Med Biol Res 40:769-778

82. Pan YK et al (2017) Acclimation to prolonged hypoxia alters hemoglobin isoform expression and increases hemoglobin oxygen affinity and aerobic performance in a marine fish. Sci Rep 7(1):7834

83. Goessling W, Sadler KC (2015) Zebrafish: an important tool for liver disease research. Gastroenterology 149(6):1361-1377

84. Hill AJ et al (2005) Zebrafish as a model vertebrate for investigating chemical toxicity. Toxicol Sci 86(1):6-19

85. Menke A et al (2011) Normal anatomy and histology of the adult zebrafish. Toxicol Pathol 39:759-775

86. Klüver N et al (2014) Transient overexpression of adh8a increases allyl alcohol toxicity in zebrafish embryos. PLoS ONE 9(3):e90619-e90619

87. Timme-Laragy AR et al (2007) Synergistic induction of AHR regulated genes in developmental toxicity from co-exposure to two model PAHs in zebrafish. Aquat Toxicol 85(4):241-250

88. Chu J, Sadler KC (2009b) New school in liver development: lessons from zebrafish. Hepatology 50(5):1656-1663

89. Jones KS et al (2008) A high throughput live transparent animal bioassay to identify non-toxic small molecules or genes that regulate vertebrate fat metabolism for obesity drug development. Nutr Metab (Lond) 5:23

90. Huang J et al (2013) A zebrafish model of conditional targeted podocyte ablation and regeneration. Kidney Int 83(6):1193-1200

91. Vliegenthart ADB et al (2014) Zebrafish as model organisms for studying drug-induced liver injury. Br J Clin Pharmacol 78(6):1217-1227

92. Quinlivan VH, Farber SA (2017) Lipid uptake, metabolism, and transport in the larval zebrafish. Front Endocrinol 8:319-319

93. Zhang X, Li C, Gong Z (2014) Development of a convenient in vivo hepatotoxin assay using a transgenic zebrafish line with liver-specific DsRed expression. PLoS One 9(3):e91874

\section{Publisher's Note}

Springer Nature remains neutral with regard to jurisdictional claims in published maps and institutional affiliations.

\section{Submit your manuscript to a SpringerOpen ${ }^{\odot}$ journal and benefit from:}

- Convenient online submission

- Rigorous peer review

- Open access: articles freely available online

- High visibility within the field

- Retaining the copyright to your article

Submit your next manuscript at springeropen.com 